\title{
Inflating the Cube by Shrinking
}

\author{
Kevin Buchin \\ buchin@inf.fu-berlin.de
}

\author{
André Schulz \\ schulza@inf.fu-berlin.de
}

Institute of Computer Science, Freie Universität Berlin, Takustr. 9, 14195 Berlin

\begin{abstract}
We present a continuous submetric deformation of the surface of the cube which increases the enclosed volume by about $25.67 \%$.

Categories and Subject Descriptors: I.3.5 [Computational Geometry and Object Modeling]

General Terms: Theory

Keywords: Submetric embeddings, surface deformation
\end{abstract}

\section{INTRODUCTION}

Every polyhedral surface in $\mathbb{R}^{3}$ has a volume-increasing isometric deformation [7]. However little is known about isometric embeddings maximizing the volume. For the polyhedra typically studied, i.e., platonic solids and doubly covered two-dimensional shapes, there is a large gap between the lower and upper bound for the maximum enclosed volume. We address the problem of how large the volume of a body with a surface isometric to that of a cube can be.

An embedding $h: S \rightarrow \mathbb{R}^{3}$ of a surface $S$ in $\mathbb{R}^{3}$ is isometric (resp. submetric) if the length of any rectifiable curve in $S$ is constant (resp. non-increasing) under $h$. An isometric (resp. submetric) deformation is a continuous map $H: S \times\left[0, \varepsilon_{0}\right] \rightarrow \mathbb{R}^{3}$ such that $h_{\varepsilon}(\cdot):=H(\cdot, \varepsilon)$ is an isometric (resp. submetric) embedding for all $0 \leq \varepsilon \leq \varepsilon_{0}$. Instead of isometric embeddings, submetric embeddings can be used since every submetric embedding of a polyhedral surface has an isometric embedding arbitrary close to it $[4,7]$.

The idea of considering volume-increasing isometric embeddings is due to Bleecker [2]. He proves that a volumeincreasing continuous isometric deformation exists for every simplicial convex surface in $\mathbb{R}^{3}$. By Alexandrov's uniqueness theorem [1] a body resulting from such a deformation must be non-convex. By Bellows conjecture (proved in [5]) the deformation does not preserve the faces of the polytope.

Recently, Pak [8] gave an easy construction for increasing the volume of the unit cube to about 1.1812 based on the work of Milka [6]. A more involved construction of Bleecker [2] yields a volume of about 1.2187. A simple upper bound of about 1.3820 can be obtained by the volume of the sphere which has the same surface area as the cube.

In this paper, we present a shrinking, i.e., a continuous, submetric deformation of the unit cube for which the resulting volume is at least 1.2567 . We first present a simple construction which we then refine. This also improves the

Copyright is held by the author/owner(s).

SCG'07, June 6-8, 2007, Gyeongju, South Korea.

ACM 978-1-59593-705-6/07/0006. lower bound on the volume of a surface isometric to that of a unit cube. Detailed calculations for the constructions in this paper can be found in [3]. The idea of looking at shrinkings in order to get isometric embeddings is due to Pak [7].

\section{FIRST CONSTRUCTION}

We present volume-increasing submetric embeddings of the cube. The embeddings are parametrized by $\varepsilon \in[0,0.5]$. Increasing $\varepsilon$ from 0 yields a continuous deformation. We first present a construction with volume about 1.2444, which we improve in the next section.

Our approach is a refinement of Igor Pak's work $[7,8]$. The original cube is given as the convex hull of the set $\{0,1\}^{3}$. We denote vertices on the surface of the cube by $p_{i}$. The same vertex in the deformed cube is denoted as $v_{i}$.
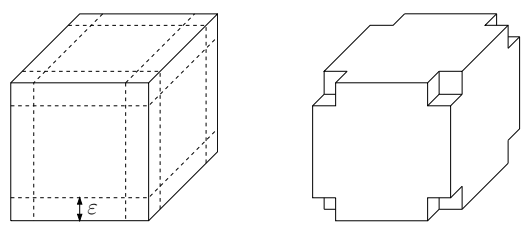

Figure 1: Cutting off $\varepsilon$ cubes.

First we cut off $\varepsilon$-cubes at every corner of the cube (see Figure 1). Next we deform the remaining part of the cube. We place one vertex in the middle of every $\varepsilon$ segment (see Figure 2.a). The segments between $p_{1}, p_{5 / 4}, p_{3 / 2}, p_{7 / 4}, p_{2}$ have the length $\varepsilon / 2$. Let the framework induced by this chain be $C$. We move the vertices of $C$ such that $v_{1}, \ldots, v_{2}$ lie on a quarter-circle (see Figure 2.b). We apply the deformation for all corresponding pairs of $\varepsilon$ segments. This leads to a body that we divide into a corpus and 12 bars. The 8

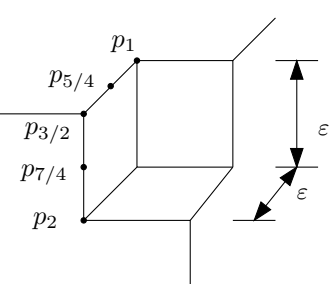

a

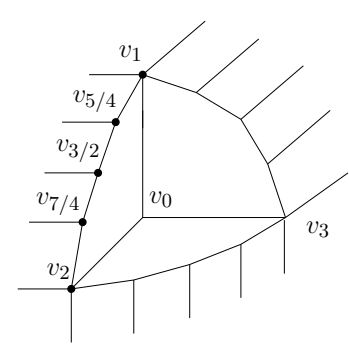

$\mathrm{b}$
Figure 2: Bending the chains induced by a pair of $\varepsilon$ segments. 

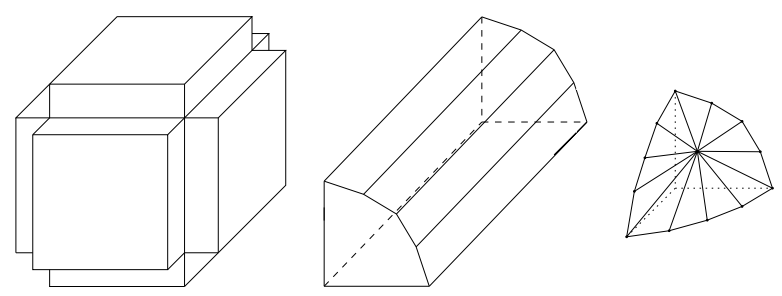

Figure 3: Corpus and bar and star.

cut off parts are called stars. Figure 3 shows the deformed parts. After the deformation a bar is a prism with a 6-gon as base area. The 6 -gon is inscribed in quarter circle. Its shorter edges have the length $\varepsilon / 2$. The radius of the quarter circle is denoted by $\delta$. The volume of one bar is given by

$$
V_{\text {bar }}=2(1-2 \varepsilon) \delta^{2} \sin (\pi / 8) .
$$

The corpus is the remaining part after cutting off the bars. Its volume is given by

$$
V_{\text {corpus }}=(1-2 \varepsilon)^{3}+6 \delta(1-2 \varepsilon)^{2} .
$$

It remains to place the deformed stars at the corners of the body. We have to deform the $\varepsilon$-cubes, such that they fit into the open 12-gons formed by three chains $C$ of the body. Consider the open part depicted in Figure 2.b. The deformed star is the convex hull of the vertices on the chains between $v_{1}, v_{2}, v_{3}$ together with $v_{0}$ and a vertex $v_{*}$. The coordinate of $v_{*}$ is chosen in such a way, that the embedding is submetric. We place $v_{*}$ on a line given by $x=y=z$. This yields coordinates $v_{*}=(0.9764 \varepsilon, 0.9764 \varepsilon, 0.9764 \varepsilon)$.

Finally, we have to evaluate the volume of the stars. Each star is divided into tetrahedra. There are two types of tetrahedra, one is given by the convex hull of $v_{0}, v_{1}, v_{5 / 4}, v_{*}$ and the other by the convex hull of $v_{0}, v_{5 / 4}, v_{3 / 2}, v_{*}$. Both tetrahedra appear 6 times in every star. That leads to a volume of a star of

$$
V_{\text {star }}=1.227259706 \varepsilon^{3} .
$$

Now we can evaluate the volume of the complete body which is

$$
V=V_{\text {corpus }}+12 V_{\text {bar }}+8 V_{\text {star }} .
$$

See Figure 4 for the graph of $V(\varepsilon)$ for feasible values of $\varepsilon$.

The volume $V(\varepsilon)$ is maximized at about $\varepsilon_{0}=0.351311$, which yields a volume $V\left(\varepsilon_{0}\right)=1.2444$. This improves the bound of Bleecker [2]. The deformed cube for this value of $\varepsilon$ is shown in Figure 5. Each star has 3 concave edges depicted as dashed lines.

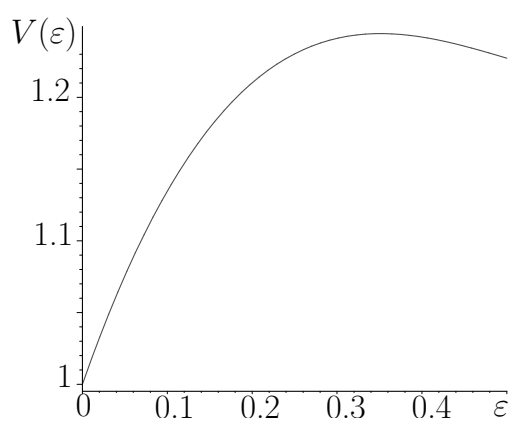

Figure 4: The volume of the deformed cube.

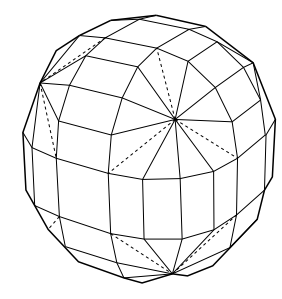

Figure 5: Deformed cube.

\section{A REFINED CONSTRUCTION}

We refine the construction to increase the volume of the cube. A crucial part of the construction was to take two adjacent edges of length $\varepsilon$ and turn them into a chain of 4 edges of length $\varepsilon / 2$. The deformation puts all vertices of the chain on a quarter circle with radius $\delta$. In the previous section the chain $C$ contained 5 vertices. If we increase the number of vertices on $C$ the deformed cube becomes more "spherish", promising a larger volume. In the limit $C$ is a spherical arc. In the following, we consider this situation.

The value of $\delta$ is the radius of a circle with perimeter $8 \pi$, thus $\delta=4 \varepsilon \pi$. The volume of the corpus is the same as calculated in Section 2. Every bar is a prism with a quarter circle of radius $\delta$ as base area. This leads to

$$
V_{\text {bar }}=(1-2 \varepsilon) \pi \delta^{2} / 4 \text {. }
$$

The stars consist of three equally sized quarter cones. The base area coincides with the base area of the bars. The height of the quarter cones is given by $a$. The value of $a$ has to be chosen in such a way that the embedding is submetric.

As feasible height we get $a=0.9772 \varepsilon$. We get

$$
V_{\text {star }}=0.9772 \varepsilon \delta^{2} \pi / 4 \text {. }
$$

Maximizing the volume of the whole body ( 1 corpus, 12 bars, 8 stars) over $\varepsilon \in[0,0.5]$ leads to a volume of 1.2567 obtained at about $\varepsilon=0.37712$.

\section{Acknowledgments}

We would like to thank Igor Pak for helpful comments and Günter Rote for suggesting the problem to us.

\section{REFERENCES}

[1] A. D. Alexandrov. Convex polyhedra. Springer Monographs in Mathematics. Springer, 2005. Original Russian edition published by Gosudarstv. Izdat. Tekhn.-Teor. Lit., Moscow-Leningrad, 1950.

[2] D. D. Bleecker. Volume increasing isometric deformations of convex polyhedra. J. Diff. Geom., 43(3):505-526, 1996.

[3] K. Buchin, I. Pak, and A. Schulz. Inflating the cube by shrinking. In Proc. 23rd European Workshop on Computational Geometry, 2007.

[4] Y. D. Burago and V. A. Zalgaller. Isometric piecewise-linear embeddings of two-dimensional manifolds with a polyhedral metric into $\mathbf{R}^{3}$. St. Petersburg Math. J., 7:369-385, 1996.

[5] R. Connelly, I. Sabitov, and A. Walz. The bellows conjecture. Contrib. Algebra Geom., 38(1):1-10, 1997.

[6] A. D. Milka. Linear bendings of regular convex polyhedra (in russian. Mat. Fiz. Anal. Geom., 1:116-130, 1994.

[7] I. Pak. Inflating polyhedral surfaces. Preprint, Department of Mathematics, MIT, 2006. $37 \mathrm{pp}$.

[8] I. Pak. Inflating the cube without stretching. arXiv:math.MG/0607754, 2006. 3 pp. to appear in Amer. Math. Monthly. 